\title{
Angiotensin-II Induced Reactive Oxygen Species: Implications in Neurogenic Hypertension
}

\author{
LU Chengzhi ${ }^{1 *}$, WANG Liं ${ }^{2}$, Aubdool-Essackjee ${ }^{2}$, Nima Sherpa² and LUO Di ${ }^{2}$
}

${ }^{1}$ Department of Cardiology, Tianjin First Central Hospital, Tianjin, China

${ }^{2}$ The Institute of Graduate, Tianjin Medical University, Tianjin, China

\begin{abstract}
Reactive oxygen species (ROS) play an important role in the development and maintenance cardio vascular diseases, especially hypertension. The relationship between ROS and hypertension has been demonstrated in several models of experimental hypertension. Accumulating evidence has suggested that the key mechanism through which Angiotensin II (Ang II) influences blood pressure is via its ability to activate ROS signaling pathways. Ang II, by stimulating angiotensin AT1 receptors, involved in the activation of $\mathrm{NAD}(\mathrm{P}) \mathrm{H}$ oxidase, which is a major source of ROS production. Despite many elegant studies that have been accumulated regarding ROS/Ang II signaling, there is still much to be elucidated in the role of ROS in neurogenic hypertension. The present review mainly discussed some recent findings documenting about the signaling mechanisms of Ang II-induced ROS in neurogenic hypertension and therapeutic target.
\end{abstract}

Keywords: Reactive Oxygen Species (ROS), Angiotensin II, Neurogenic hypertension

Abbreviations: Ang II: Angiotensin II; AT1R: Angiotensin Type 1 Receptor; BDNF: Brain-Derived Neurotrophic Factor; CREB: cAMP Response Element Binding Protein; MAP: Mean Arterial Pressure; $\mathrm{O}_{2}^{\bullet}:$ Superoxide Anion; ROS: Reactive Oxygen Species; RVLM: Rostral Ventrolateral Medulla; SOD: Superoxide Dismutase; SHR: Spontaneously Hypertensive Rat; TrkB: Tropomyosin receptor kinase B; WKY: Wistar-Kyoto; UCP: Uncoupling Protein; BBB: Blood Brain Barrier ; CaMKII: Calmodulin Kinase II; MAPK: Mitogen-Activated Protein Kinase; TF: Transcriptional Factors; NFkB: Nuclear Factor$\kappa \mathrm{B}$; TH: Tyrosine Hydroxylase; AP-1: Activator Protein-1; 2K1C: Two Kidney One Clip; RVH: Renovascular Hypertension; ESRK: Extracellular Signal-Regulated Kinase; RSK: ribosomal Protein S6 Kinase; COX: Cyclooxygenase; PGE: Prostaglandin E2; IP3: Inositol Triphosphate; SFO: Subfornical Organ; PVN: Paraventricular Nucleus; NTS: Nucleus Tractus Solitaries; ETC: Electron Transport Capacity

\section{Introduction}

Hypertension is the most important risk factor for cardio- vascular disease and, thus, remains a global public health challenge. Despite lots of advances in antihypertensive agents, it has been extremely difficult to manage hypertension in $40 \%$ of hypertensive patients [1]. A majority of unresponsive patients may have neurogenic hypertension which is associated with a rise in sympathetic outflow and often, an inhibition of parasympathetic drive [2-4].

Many investigators demonstrated that neurogenic hypertension is caused in part by the formation of Ang II-induced ROS [5-11]. However, since Ang II is composed by eight amino acids, which make it incapable of crossing the Blood Brain Barrier (BBB). As a result, locally produced Ang II may play a key role for brain ROS. In central neurons, local angiotensin II (Ang II) activates the Angiotensin II Type 1 Receptor (AT1R) to induce a cascade of intra-neuronal signaling events that ultimately leads to changes in membrane potential and an increase in neuronal firing [6]. Accumulating evidence over the past years has established that Ang II increases levels of ROS, particularly $\mathrm{O}_{2}{ }^{--}$, in neurons which contribute to the increase in neuronal activation [7-12].

As we all known, there is a large amount of signaling pathways in the upstream and downstream of ROS, some of which is familiar to many researchers, some of which is newly discovered, some of which is a protective role. Next, I will give a brief introduction of ROS-mediated signaling pathway.

\section{NADPH-ROS signaling pathway}

Basically, it is the most well known signaling pathway, confirmed by many investigators. Ang II is upstream of NADPH oxidase activation, which requires Rac1 [13-15], a regulator of Nox1 and Nox2. Adenoviral-mediated inhibition of Racl decreases BP. Abundant evidence suggests that a key mechanism by which Ang II influences blood pressure is via its ability to stimulate the production of ROS, mainly superoxide anion, via activation of NADPH oxidase [16-19]. A recent study demonstrated increased expression of three isoforms of NADPH oxidase (i.e. Nox1, Nox 2 and Nox 4) and enzyme activity in Spontaneously Hypertensive Rat (SHR) [20], but not in nomortensive rat. NADPH oxidase, an enzyme composed of two membrane-bound subunits (gp91phox and p22phox), cytoplasmic subunits (p40phox, p47phox and p67phox) and the small G-protein Racla [21], is a major source of ROS in hypertension $[21,22]$ and has a critical role in generating ROS in the brain [14,22-25].

The general process of this signaling pathway: Firstly, Ang II activates the AT1 receptors, then, the cytoplasmic subunits bind to the membrane subunits and activate the enzyme, resulting in the intracellular production of superoxide (converts the intracellular nitric oxide to peroxynitrite). The accumulation of superoxide leads to changes in ion channels, particularly calcium and potassium channels, altering neuronal firing properties in areas of the brain such as the

*Corresponding author: Dr. LU Cheng-zhi, Department of Cardiology, Tianjin First Central Hospital, Tianjin China,24, Fukang Road, Nankai District, Tianjin, 300192 China, Tel: +86-022-23626585; Fax: +86-022-23626585; E-mail: lucz8@126.com

Received June 12, 2012; Accepted July 17, 2012; Published July 20, 2012

Citation: Chengzhi LU, Li W, Essackjee A, Sherpa N, Di LUO (2012) AngiotensinII Induced Reactive Oxygen Species: Implications in Neurogenic Hypertension. J Hypertens 1:106. doi:10.4172/2167-1095.1000106

Copyright: (c) 2012 Chengzhi LU, et al. This is an open-access article distributed under the terms of the Creative Commons Attribution License, which permits unrestricted use, distribution, and reproduction in any medium, provided the original author and source are credited. 
RVLM, resulting in increased sympathetic nerve activity and increased blood pressure [26] (Figure 1).

Yin et al. [10], recently reported that Ang II increases mitochondrial $\mathrm{O}_{2}{ }^{--}$levels in catecholaminergic (CATH.a) neurons and that this increase in mitochondrial $\mathrm{O}_{2}^{--}$mediates the Ang II-induced activation of a redox-sensitive kinase, calcium/calmodulin kinase II (CaMKII), and the Ang II-induced inhibition of neuronal potassium current (Ik). In addition, increase in concentration of intracellular calcium also result in activation of the CaMKII complex. CaMKII act on ion channels in the cell membrane changing neuronal firing and induced hypertension.

\section{MAPK-AP1/ NFkB pathway}

Ang-II also activates the Mitogen-Activated Protein Kinase (MAPK) pathway. ROS directly activate the MAPK, which increases the NADPH oxidase activity leading to more ROS. MAPK also activates nuclear Transcriptional Factors (TF), such as NFkB and AP1 , which modulate the expression of Tyrosine Hydroxylase $(\mathrm{TH})$ and AT1 receptors (Figure 2).

In hypertension, angiotensin II (Ang II) and ROS can increase the activity of the transcription factor Nuclear Factor- $\kappa B$ $(\mathrm{NF \kappa B})$, which, in turn, can further increase ROS expression in a positive feed-forward way [27-31], and modulate the expression of Tyrosine Hydroxylase (TH) and AT1 receptors. Along with Ang II, NFkB acts to increase the presence of ROS, such as $\mathrm{O}_{2}$ , which subsequently affects the present NO levels, thereby effecting neuronal activity/function and NE release. Blockade of NFKB at two separate locations in its activation pathway prevents these changes, restores the RAS balance, and promotes the antihypertensive RAS arm, including ACE2 and the MasR. This blockage also reduces ROS expression and elevates nNOS, all of which contribute to a reduction in sympathetic outflow and an improvement in the Ang II-induced hypertensive state [32]

The other downstream mechanisms by which ROS affect the PVN neurons is by modulating activator protein-1 (AP-1) activity [33]. AP-1 activity can be longitudinally monitored in vivo by bioluminescence imaging in $2 \mathrm{~K} 1 \mathrm{C}$ or sham mice model that had undergone PVNtargeted microinjections of an adenovirus encoding the firefly luciferase (Luc) gene downstream of AP-1 response elements (AdAP-1Luc) [34]. In 2K1C-induced Renovascular Hypertension (RVH) model in mice, Burmeister et al. [33] found that AP-1 is robustly activated in the PVN with a time-course that is consistent with its involvement in the development of hypertension, and its inhibition by $\mathrm{O}_{2}{ }^{-}$scavenging parallels the inhibition of hypertension. And they also found that dominant-negative inhibition of AP-1 transcriptional activity in the PVN prevents RVH. Therefore they concluded that RVH is mediated by oxidative stress-induced AP-1 activation in the PVN.

\section{The ERK1/2-RSK-nNOS Signaling Pathway}

Previous study showed that eNOS might play a critical role in NTS cardiovascular function via the adenosine- extracellular signal-regulated kinase (ERK)1/2- eNOS signaling pathway [35]. But recently, Wen et al. [36] found nNOS increased after inhibition of Ang II downstream of AT $\mathrm{R}$ or depletion of ROS in the NTS of $\mathrm{SHR}_{\mathrm{S}}$ which suggests nNOS might be one of the downstream targets of Ang II that is involved in NO production and that modulates blood pressure function in the NTS of SHRs. They also observed that ROS accumulation inhibited ERK1/2 activation in the NTS and induced hypertension. Wu et al. [37] demonstrated that ROS can enhance protein tyrosine kinase activity to regulate extracellular signal-regulated kinase (ERK1/2 phosphorylation in neuroblastoma in vitro). Chen et al. [38] also reported that activation of protein phosphatases (e.g. PP2A or PP5) can inhibit hydrogen peroxide-induced ERK1/2 phosphorylation in neuronal cells. ERK1/2 is involved in cellular differentiation, proliferation and development in neuronal cells. Thus, ERK1/2 not only regulates neuronal development but also play regulatory roles in neurons in response to various physiological stimuli.

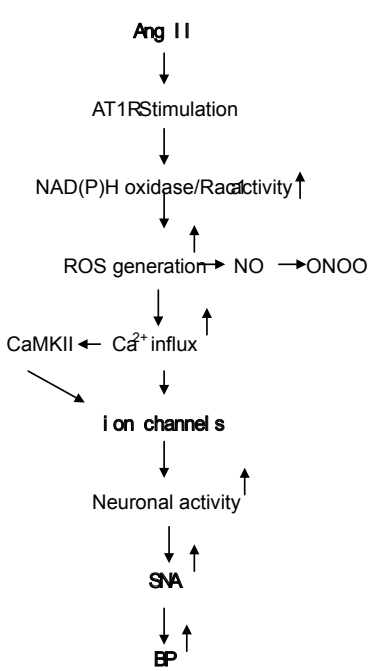

Figure 1: NADPH-ROS Signaling Pathway

The general process of this signaling pathway: firstly, Ang II activates the AT1 receptors, then, the cytoplasmic subunits bind to the membrane subunits and activate the enzyme, resulting in the intracellular production of superoxide(converts the intracellular nitric oxide to peroxynitrite). The accumulation of superoxide leads to changes in ion channels, particularly calcium and potassium channels, altering neuronal firing properties in areas of the brain such as the RVLM, resulting in increased sympathetic nerve activity and increased blood pressure [27]. In addition, the intracellular calcium concentration increased also result in activation of the CaMKII complex CaMKII act on ion channels in the cell membrane changing neuronal firing and induced hypertension.

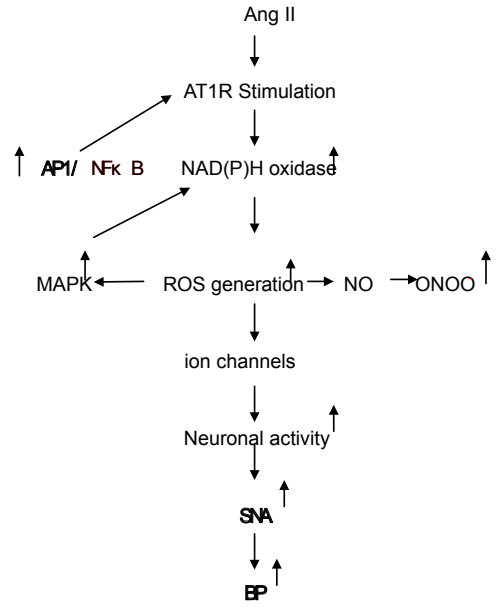

Figure 2: MAPK-AP1/ NFKB pathway

Ang-II activates the Mitogen-Activated Protein Kinase (MAPK) pathway. ROS directly activate the MAPK, which increases the NADPH oxidase activity leading to more ROS. MAPK also activates nuclear Transcriptional Factors (TF), such as NFKB and AP-1, which modulate the expression of Tyrosine Hydroxylase (TH) and AT1 receptors. 
In conclusion, Ang II binds to the AT1R, leading to superoxide production. Superoxide accumulation may inhibit ERK1/2 activity. Moreover, Ribosomal protein S6 Kinase (RSK) and nNOS cascade activity is also inhibited. This ultimately leads to decreased NO concentrations in the NTS and increased blood pressure. Ang II may inhibit ERK1/2-RSK-nNOS signaling, (Figure 3) not only through ROS but also through other signaling molecules, to modulate blood pressure in the NTS of SHRs.

\section{COX-1-derived $\mathrm{PGE}_{2}$ and $\mathrm{EP}_{1} \mathrm{R}$ signal pathway}

Cyclooxygenase (COX)-derived prostanoid signaling has long been implicated in the pathogenesis of Ang II-dependent hypertension. Ang II-evoked ROS formation in dissociated SFO cells is prevented by inhibition of COX-1 but not COX-2. A key finding of the present study demonstrates that COX-1-derived prostaglandin E2 (PGE2) and its receptor EP1 (EP1R) are required for this Ang II-evoked ROS formation in the SFO in vitro and in vivo [39]. And both in vitro and in vivo inhibition of EP1R prevent Ang II-induced ROS accumulation in the SFO, a response that is known to have a causative role in slowpressor Ang II hypertension [12,40]. Virally mediated reconstitution of EP1R selectively in the SFO of EP1R-null mice restores hypertension and SFO ROS formation in response to slow pressor Ang II infusions. It is the first evidence that COX-1-derived PGE2/EP1R signaling in the SFO is required for the ROS-mediated hypertension elicited in the slow-pressor Ang II model. It is a key role for PGE2/EP1R signaling in the CNS, particularly the SFO, in mediating systemic Ang II-dependent hypertension [39].

NADPH oxidase mediates PGE2/EP1R-mediated ROS formation in the SFO may be through $\mathrm{Ca}^{2+}$ signaling. Because activation of EP1R results in inositol triphosphate (IP3)-mediated release of intracellular $\mathrm{Ca}^{2+}$ and reduced $\mathrm{Ca}^{2+}$ efflux through the $\mathrm{Na}^{+} / \mathrm{Ca}^{2+}$ exchanger [41].

\section{BDNF/TrkB-UCP2 signaling pathway (protective pathway)}

One of the well-recognized protective mechanisms against stressful insults in brain is expression of neurotrophic factors, in particular Brain-Derived Neurotrophic Factor (BDNF) [42]. BDNF engaged in oxidative stress-associated neurogenic hypertension via negativefeedback regulation of tissue $\mathrm{O}_{2}^{-}$levels in RVLM, protects striatal neurons from cell death as an antioxidant [42]. Ang II induces $\mathrm{O}^{2-}$ dependent upregulation of BDNF in RVLM via phosphorylation of cAMP response element binding protein (CREB). The Ang II-activated $\mathrm{BDNF} / \mathrm{TrkB}$ signaling, in turn, exerts negative-feedback regulation on tissue $\mathrm{O}^{2-}$ level in RVLM [43]. BDNF and the Tropomyosin receptor kinase (Trk) B are distributed in brainstem nuclei that subserve neural regulation of arterial pressure $[44,45]$.

Recently, Chan et al. [46] suggested that transcriptional upregulation of mitochondrial uncoupling protein 2 (UCP2) in response to an increase in superoxide plays an active role in the feedback regulation of ROS production in the RVLM. BDNF restores the reduced mitochondrial electron transport capacity (ETC) and upregulates mitochondrial UCP2 expression in RVLM. UCP2 is a homolog of UCP protein family of mitochondrial anion transporters that uncouple ATP synthesis from oxidative phosphorylation by causing proton leakage across the mitochondrial inner membrane, leading to a decrease in proton electrochemical gradient across the inner mitochondrial membrane and the resultant mitigation in the production of mitochondria-derived ROS, in particular $\mathrm{O}_{2}$. Through inhibition of $\mathrm{p} 47$ phoxphosphorylation, preservation of mitochondrial electron transport capacity, and upregulation of mitochondrial UCP2, result in protection against Ang II-induced hypertension and oxidative stress. Besides, stimulation of PPAR- $\gamma$ also results in the upregulation of UCP2, thereby ameliorates Ang II-induced chronic oxidative stress and long-term pressor response (Figure 4).

\section{Other signaling pathway}

Except the signaling pathway above, there is another pathway may involve in the Ang II induced hypertension. Such as Kirabo A et al. [47] found that, vascular smooth muscle cells (VSMCs) Jak2 expression is involved in the pathogenesis of Ang II-dependent hypertension due to the increased presence of reactive oxygen species (ROS). Chan et al. [48] reported that, in neurons, damaged ETC complexes are a source of mitochondrial-produced ROS (Figure 5). Though there have been many signaling pathway were found, additional studies are still required to examine the mechanism(s) by which ROS increase sympathetic tone and drive the development of hypertension.

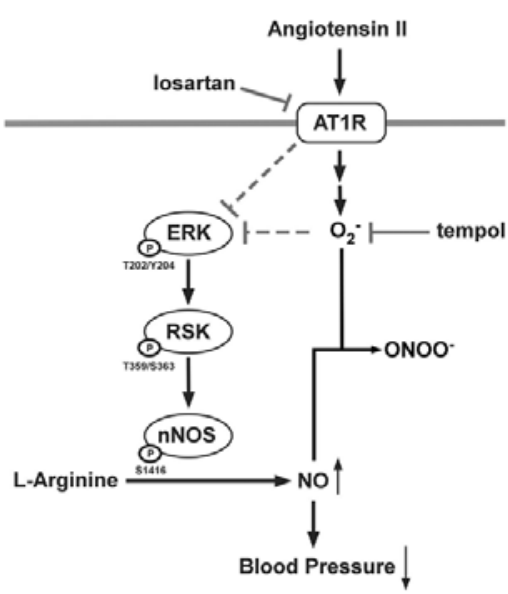

Figure 3: The ERK1/2-RSK-nNOS Signaling Pathway

Cheng WH, Lu PJ, et al [39] Angiotensin II Inhibits Neuronal Nitric Oxide Synthase Activation through the ERK1/2-RSK Signaling Pathway to Modulate Central Control of Blood Pressure.

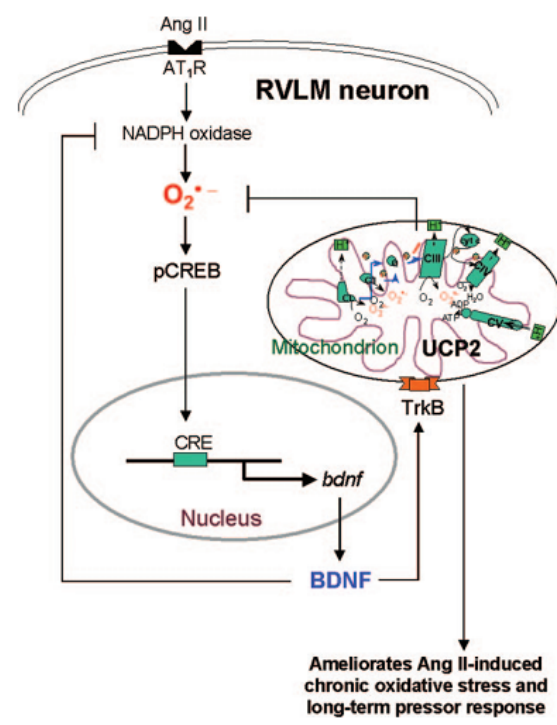

Figure 4: BDNF/TrkB-UCP2 signaling pathway

Samuel H.H. Chan, Chih-Wei J et al. [46] Transcriptional Upregulation of BrainDerived Neurotrophic Factor in Rostral Ventrolateral Medulla by Angiotensin II. 


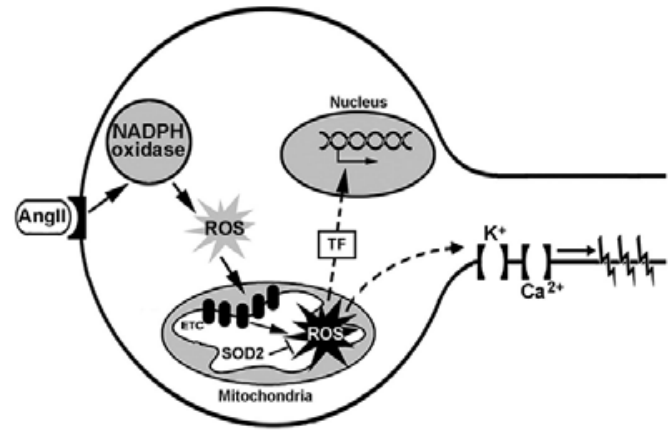

Figure 5: Ang II stimulation of neurons in the RVLM increases NADPH oxidase-derived ROS, which, in turn, damage mitochondrial ETC complexes leading to an increase in mitochondrial-produced ROS

\section{The Novel Therapeutic Targets of Neurogenic Hyper- tension}

ROS have been shown as important signaling factors for enhancement of the neurogenic hypertensive response through Ang II mechanisms [12,30,31]. Thus, potentially inhibiting one of the mechanistic pathways by which the hypertensive response and sympathoexcitation, is modulated. These conclusions are confirmed by previously that ICV administration of the ROS scavenger, Tempolan SOD mimetic, reduced renal sympathetic nerve activity in Ang II-treated rats. Similarly, acute inhibtion of the NADPH oxidase with apocynin or scavenging of superoxide with tiron, a superoxide dismutase mimetic, improves the baroreflex sensitivity in spontaneously hypertensive rats [49]. In addition, microinjection of vitamin $\mathrm{C}$ into the PVN also reduces the high blood pressure and sympathetic activity of renovascular hypertensive rats $[50,51]$.

Of note growing evidence now suggests that hypertension and sympathoexcitation can be prevented /attenuated by selective genetic or pharmacological manipulation of AT1 mRNA expression specifically in the SFO or RVLM. In support of that concept, Zimmerman et al. [12] have shown that intracerebroventricular injection of losartan, an AT1 receptor antagonist targeting the SFO, prevents the hypertension induced by chronic peripheral ang II infusion in mice. Administration of losartan reduces ROS accumulation in the RVLM and improves baroreflex sensitivity.

Considering the central position that MAPK plays in regulating the neurogenic component of hypertension, choline exerts significant antihypertensive activity through inhibition of ROS-mediated p38 MAPK activation as well as regulation of $\mathrm{Ca}^{2+}$-mediated calcineurin cell signal transduction pathway. Rosiglitazone also be able to inhibit Ang IIinduced CRP generation in HAECs by regulating AT(1)-ROS-MAPK signal pathway. And bilateral $\mathrm{NF \kappa B}$ (the downstream of MAPK) blockade can reduce the ROS reactions typically associated with Ang II-induced hypertension [32].

In addition, EP1R, as part of signaling pathway, is a novel target for the treatment of hypertension. Pharmacological inhibition of EP1R selectively in the CNS prevents slow-pressor Ang II hypertension, and central Ang II-driven sympathetic and dipsogenic responses are also mediated by brain EPR [39].

Direct RVLM administration of coenzyme Q10, a mitochondrial electron transporter and antioxidant, restored electron transport capacity, decreased mitochondrial- localized ROS production, and significantly reduced mean systemic arterial pressure and sympathetic neurogenic vasomotor tone in SHRs [52]. Therefore, Coenzyme Q10 can be used as a means of treatment of hypertension.

Intra-neuronal signaling and the downstream redox-sensitive proteins involved in controlling the neuronal discharge rate, the sympathetic outflow. Thus, the role of elevated sympathetic nervous system in neurogenic hypertension becomes an important therapeutic target, and corroborated by the recent successes in its treatment by renal sympathetic denervation in humans [53-56]. In our laboratory we found that [56], in mongrel neurogenic hypertensive dogs, renal Sympathetic Denervation causes substantial and sustained blood pressure reduction, along with reducing the level of angiotensin II and increasing the expression of ACE2. Moreover, in our clinical trials of resistant hypertension, we also found the similar results.

Future studies are needed to identify new redox-based therapeutics. Upregulation of endogenous antioxidants in the regulation of ROS homeostasis and renal sympathetic denervation are both potential therapeutic targets.

\section{Summary and Conclusion}

In summary, abundant research now suggests that a key mechanism by which Ang-II influences blood pressure is via its ability to activate ROS signaling pathways. Upstream and downstream consequences of the precise mechanisms are discussed. Several Questions remain, however, because the ROS signaling pathway are complex. Further studies are required to gain a better understanding of the role of brain ROS in autonomic cardiovascular regulation and potential therapeutic targets.

\section{References}

1. Primatesta P, Brookes M, Poulter NR (2001) Improved hypertension management and control: results from the health survey for England 1998. Hypertension 38: 827-832.

2. Mann SJ (2003) Neurogenic essential hypertension revisited: the case for increased clinical and research attention. Am J Hypertens 16: 881-888.

3. Grassi G, Quarti-Trevano F, Brambilla G, Seravalle G (2010) Blood pressure control in resistant hypertension: new therapeutic options. Expert Rev Cardiovasc Ther 8: 1579-1585.

4. Fisher JP, Fadel PJ (2010) Therapeutic strategies for targeting excessive central sympathetic activation in human hypertension. Exp Physiol 95: 572580 .

5. Zimmerman MC, Lazartigues E, Lang JA, Sinnayah P, Ahmad IM, et al. (2002) Superoxide mediates the actions of angiotensin II in the central nervous system. Circ Res 91: 1038-1045.

6. Sumners C, Fleegal MA, Zhu M (2002) Angiotensin AT1 receptor signalling pathways in neurons. Clin Exp Pharmacol Physiol 29: 483-490.

7. Sun C, Sellers KW, Sumners C, Raizada MK (2005) NAD(P)H oxidase inhibition attenuates neuronal chronotropic actions of angiotensin II. Circ Res 96: $659-666$

8. Wang G, Anrather J, Huang J, Speth RC, Pickel VM, et al. (2004) NADPH oxidase contributes to angiotensin II signaling in the nucleus tractus solitarius. J Neurosci 24: 5516-5524.

9. Wang G, Anrather J, Glass MJ, Tarsitano MJ, Zhou P, et al. (2006) Nox2 $\mathrm{Ca} 2+$, and protein kinase $\mathrm{C}$ play a role in angiotensin II-induced free radical production in nucleus tractus solitarius. Hypertension 48: 482-489.

10. Yin JX, Yang RF, Li S, Renshaw AO, Li YL, et al. (2010) Mitochondria-produced superoxide mediates angiotensin II-induced inhibition of neuronal potassium current. Am J Physiol Cell Physiol 298: C857-865.

11. Zimmerman MC, Lazartigues E, Sharma RV, Davisson RL (2004) Hypertension caused by angiotensin II infusion involves increased superoxide production in the central nervous system. Circ Res 95: 210-216.

12. Zimmerman MC, Sharma RV, Davisson RL (2005) Superoxide mediates 
Citation: Chengzhi LU, Li W, Essackjee A, Sherpa N, Di LUO (2012) Angiotensin-II Induced Reactive Oxygen Species: Implications in Neurogenic Hypertension. J Hypertens 1:106. doi:10.4172/2167-1095.1000106

angiotensin II-induced influx of extracellular calcium in neural cells. Hypertension 45: 717-723.

13. Wang G, Anrather J, Huang J, Speth RC, Pickel VM, et al. (2004) NADPH oxidase contributes to angiotensin II signaling in the nucleus tractus solitarius. J Neurosci 24: 5516-5524.

14. Zimmerman MC, Dunlay RP, Lazartigues E, Zhang Y, Sharma RV, et al. (2004) Requirement for Rac1-dependent NADPH oxidase in the cardiovascular and dipsogenic actions of angiotensin II in the brain. Circ Res 95: 532-539.

15. Hirooka Y, Sagara Y, Kishi T, Sunagawa K (2010) Oxidative stress and centra cardiovascular regulation. - Pathogenesis of hypertension and therapeutic aspects -. Circ J 74: 827-835.

16. Braga VA, Burmeister MA, Zhou Y, Sharma RV, Davisson RL.Selective ablation of AT1a receptors in rostral ventrolateral medulla(RVLM) prevents chronic angiotensin-II-dependent hypertension in part by reducing oxidant stress in this region. Hypertension 2008;52: E34 (Abstract).

17. Griendling KK, Minieri CA, Ollerenshaw JD, Alexander RW (1994) Angiotensin II stimulates $\mathrm{NADH}$ and NADPH oxidase activity in cultured vascular smooth muscle cells. Circ Res 74: 1141-1148.

18. Berry C, Hamilton CA, Brosnan MJ, Magill FG, Berg GA, et al. (2000) Investigation into the sources of superoxide in human blood vessels: angiotensin II increases superoxide production in human internal mammary arteries. Circulation 101: 2206-2212.

19. Nunes FC, Ribeiro TP, França-Silva MS, Medeiros IA, Braga VA (2010) Superoxide scavenging in the rostral ventrolateral medulla blunts the pressor response to peripheral chemoreflex activation. Brain Res 1351: 141-149.

20. Briones AM, Tabet F, Callera GE, Montezano AC, Yogi A, et al. (2011) Differential regulation of Nox1, Nox2 and Nox4 in vascular smooth muscle cells from WKY and SHR. J Am Soc Hypertens 5: 137-153.

21. Lassègue $B$, Clempus RE (2003) Vascular NAD(P)H oxidases: specific features, expression, and regulation. Am J Physiol Regul Integr Comp Physio 285: R277-297.

22. Bai Y, Jabbari B, Ye S, Campese VM, Vaziri ND (2009) Regional expression of $\mathrm{NAD}(\mathrm{P}) \mathrm{H}$ oxidase and superoxide dismutase in the brain of rats with neurogenic hypertension. Am J Nephrol 29: 483-492.

23. Campese VM, Sindhu RK, Ye S, Bai Y, Vaziri ND, et al. (2007) Regional expression of $\mathrm{NO}$ synthase, $\mathrm{NAD}(\mathrm{P}) \mathrm{H}$ oxidase and superoxide dismutase in the rat brain. Brain Res 1134: 27-32.

24. Infanger DW, Sharma RV, Davisson RL (2006) NADPH oxidases of the brain distribution, regulation, and function. Antioxid Redox Signal 8: 1583-1596.

25. Nozoe M, Hirooka Y, Koga Y, Sagara Y, Kishi T, et al. (2007) Inhibition of Rac1-derived reactive oxygen species in nucleus tractus solitarius decreases blood pressure and heart rate in stroke-prone spontaneously hypertensive rats. Hypertension 50: 62-68.

26. Braga VA, Medeiros IA, Ribeiro TP, França-Silva MS, Botelho-Ono MS, et al (2011) Angiotensin-II-induced reactive oxygen species along the SFO-PVNRVLM pathway: implications in neurogenic hypertension. Braz J Med Biol Res 44: 871-876.

27. Kang YM, Ma Y, Zheng JP, Elks C, Sriramula S, et al. (2009) Brain nuclear factor-kappa B activation contributes to neurohumoral excitation in angiotensin II-induced hypertension. Cardiovasc Res 82: 503-512.

28. Bubici C, Papa S, Dean K, Franzoso G (2006) Mutual cross-talk between reactive oxygen species and nuclear factor-kappa $\mathrm{B}$ : molecular basis and biological significance. Oncogene 25: 6731-6748.

29. Cardinale JP, Sriramula S, Pariaut R, Guggilam A, Mariappan N, et al. (2010) HDAC inhibition attenuates inflammatory, hypertrophic, and hypertensive responses in spontaneously hypertensive rats. Hypertension 56: 437-444.

30. van den Berg R, Haenen GR, van den Berg H, Bast A (2001) Transcription factor NF-kappaB as a potential biomarker for oxidative stress. $\mathrm{Br} \mathrm{J}$ Nutr 86 121-127.

31. Zhang ZH, Wei SG, Francis J, Felder RB (2003) Cardiovascular and rena sympathetic activation by blood-borne TNF-alpha in rat: the role of central prostaglandins. Am J Physiol Regul Integr Comp Physiol 284: R916-927.

32. Cardinale JP, Sriramula S, Mariappan N, Agarwal D, Francis J (2012) Angiotensin II-induced hypertension is modulated by nuclear factor-KBin the paraventricular nucleus. Hypertension 59: 113-121.
33. Burmeister MA, Young CN, Braga VA, Butler SD, Sharma RV et al. (2011) In vivo bioluminescence imaging reveals redox-regulated activator protein-1 activation in paraventricular nucleus of mice with renovascular hypertension. Hypertension 57: 289-297.

34. Peterson JR, Infanger DW, Braga VA, Zhang Y, Sharma RV, et al. (2008) Longitudinal noninvasive monitoring of transcription factor activation in cardiovascular regulatory nuclei using bioluminescence imaging. Physio Genomics 33: 292-299.

35. Ho WY, Lu PJ, Hsiao M, Hwang HR, Tseng YC et al. (2008) Adenosine modulates cardiovascular functions through activation of extracellular signalregulated kinases 1 and 2 and endothelial nitric oxide synthase in the nucleus tractus solitarii of rats. Circulation 117: 773-780.

36. Cheng WH, Lu PJ, Ho WY, Tung CS, Cheng PW, et al. (2010) Angiotensin II inhibits neuronal nitric oxide synthase activation through the ERK1/2-RSK signaling pathway to modulate central control of blood pressure. Circ Res 106 : 788-795.

37. Griendling KK, Sorescu D, Ushio-Fukai M (2000) NAD(P)H oxidase: role in cardiovascular biology and disease. Circ Res 86: 494-501.

38. Wu H, Ichikawa S, Tani C, Zhu B, Tada M, et al. (2009) Docosahexaenoic acid induces ERK1/2 activation and neuritogenesis via intracellular reactive oxygen species production in human neuroblastoma SH-SY5Y cells. Biochim Biophys Acta 1791: 8-16.

39. Cao X, Peterson JR, Wang G, Anrather J, Young CN, et al. (2012) Angiotensin II-dependent hypertension requires cyclooxygenase 1-derived prostaglandin $\mathrm{E} 2$ and EP1 receptor signaling in the subfornical organ of the brain. Hypertension 59: 869-876

40. Lob HE, Marvar PJ, Guzik TJ, Sharma S, McCann LA, et al. (2010) Induction of hypertension and peripheral inflammation by reduction of extracellula superoxide dismutase in the central nervous system. Hypertension 55: 277 283

41. Kawano T, Anrather J, Zhou P, Park L, Wang G, et al. (2006) Prostaglandin E2 EP1 receptors: downstream effectors of COX-2 neurotoxicity. Nat Med 12 225-229.

42. Levi-Montalcini R (1987) The nerve growth factor 35 years later. Science 237 1154-1162.

43. Chan SH, Wu CW, Chang AY, Hsu KS, Chan JY (2010) Transcriptiona upregulation of brain-derived neurotrophic factor in rostral ventrolateral medulla by angiotensin II: significance in superoxide homeostasis and neural regulation of arterial pressure. Circ Res 107: 1127-1139.

44. Katoh-Semba R, Takeuchi IK, Semba R, Kato K (1997) Distribution of brainderived neurotrophic factor in rats and its changes with development in the brain J Neurochem 69: 34-42.

45. Yan Q, Radeke MJ, Matheson CR, Talvenheimo J, Welcher AA, et al. (1997) Immunocytochemical localization of TrkB in the central nervous system of the adult rat. J Comp Neurol 378: 135-157.

46. Chan SH, Wu CA, Wu KL, Ho YH, Chang AY, et al. (2009) Transcriptional upregulation of mitochondrial uncoupling protein 2 protects against oxidative stress-associated neurogenic hypertension. Circ Res 105: 886-896.

47. Kirabo A, Kearns PN, Jarajapu YP, Sasser JM, Oh SP, et al. (2011) Vascula smooth muscle Jak2 mediates angiotensin II-induced hypertension via increased levels of reactive oxygen species. Cardiovasc Res 91: 171-179.

48. Chan SH, Wu KL, Chang AY, Tai MH, Chan JY (2009) Oxidative impairmen of mitochondrial electron transport chain complexes in rostral ventrolateral medulla contributes to neurogenic hypertension. Hypertension 53: 217-227.

49. Botelho-Ono MS, Pina HV, Sousa KH, Nunes FC, Medeiros IA, et al. (2011) Acute superoxide scavenging restores depressed baroreflex sensitivity in renovascular hypertensive rats. Auton Neurosci 159: 38-44.

50. Oliveira-Sales EB, Nishi EE, Carillo BA, Boim MA, Dolnikof MS, Bergamaschi $\mathrm{CT}$, et al. Oxidative stress in the sympa thetic premotor neurons contributes to sympathetic activetion in renovascular hypertension. Am J Hypertens 2009 22 484-492.

51. Campos RR, Oliveira-Sales EB, Nishi EE, Boim MA, Dolnikoff MS, Bergamaschi CT. The role of oxidative stress in renovascular hypertension. Clin Exp Pharmacol Physio 2011; 38: 144-152.

52. Hirooka Y, Kishi T, Sakai K, Takeshita A, Sunagawa K (2011) Imbalance of 
Citation: Chengzhi LU, Li W, Essackjee A, Sherpa N, Di LUO (2012) Angiotensin-II Induced Reactive Oxygen Species: Implications in Neurogenic Hypertension. J Hypertens 1:106. doi:10.4172/2167-1095.1000106

central nitric oxide and reactive oxygen species in the regulation of sympathetic activity and neural mechanisms of hypertension. Am J Physiol Regul Integr Comp Physiol 300: R818-826.

53. Krum H, Schlaich M, Whitbourn R, Sobotka PA, Sadowski J, et al. (2009) Catheter-based renal sympathetic denervation for resistant hypertension: a multicentre safety and proof-of-principle cohort study. Lancet 373: 1275-1281.

54. Symplicity HTN-2 Investigators, Esler MD, Krum H, Sobotka PA, Schlaich MP, et al. (2010) Renal sympathetic denervation in patients with treatment- resistant hypertension (The Symplicity HTN-2 Trial): a randomised controlled trial. Lancet 376: 1903-1909.

55. Symplicity HTN-1 Investigators (2011) Catheter-based renal sympathetic denervation for resistant hypertension: durability of blood pressure reduction out to 24 months. Hypertension 57: 911-917.

56. Lu CZ, Liu J, Xia DV, Zhao XD, Chen X, et al. (2012) [Efficacy of catheterbased renal denervation in mongrel neurogenic hypertensive dogs]. Zhonghua Xin Xue Guan Bing Za Zhi 40: 14-17. 